\title{
Mathematical Model of the Pharmacokinetic Behavior of Orally Administered Erythromycin to Healthy Adult Male Volunteers
}

\author{
Mária Ďurišová* \\ Department of Pharmacology of Inflammation, Institute of Experimental Pharmacology and Toxicology, Slovak Academy of Sciences, Bratislava, \\ Dúbravská cesta 4
}

Received: September 06, 2015, Accepted: November 11, 2015, Published: December 16, 2015

*Corresponding author: Mária Ďurišová, Department of Pharmacology of Inflammation, Institute of Experimental Pharmacology and Toxicology, Slovak Academy of Sciences, Bratislava, Dúbravská cesta 4, E-mail: exfamadu@savba.sk

\begin{abstract}
Objectives: The main objective of the current study was to present a further example which showed a victorious use of an superior mathematical modeling method based on the concept of a dynamic system in mathematical modeling in pharmacokinetics. An additional objective was to motivate researchers working in field of pharmacokinetics to use of an alternative modeling method to those modeling methods commonly used in pharmacokinetic studies. The current study is a escort piece of a correlated (Yakatan et al. 1979) study in volunteers published in the Journal of Pharmacokinetics and Biopharmaceutics. In the study cited at this point, an investigation of bioequivalence of erythromycin stearate tablets in man was described.
\end{abstract}

Methods: Data published in the study cited above and an advanced modeling method were used. (For the method used, please see e.g. the explanatory picture and the full-text studies at: http:// www.uef.sav.sk/advanced.htm)

Results: All mathematical models developed successfully fitted the measured data. Based on the mathematical models developed, main pharmacokinetic variables of erythromycin were determined.

Conclusion: The mathematical modeling method used in the current study is universal, comprehensive, and flexible. Therefore, it can be used to develop mathematical models not only in pharmacokinetics but also in many other scientific fields.

Keywords: Erythromycin; Oral administration; Mathematica model

\section{Introduction}

Erythromycin is an antibiotic useful for the treatment of a number of bacterial infections. This includes respiratory tract infections, skin infections, chlamydia infections, and syphilis. It may also be used during pregnancy to prevent Group B streptococcal infection in newborns [1]. It is an effective inhibitor of CYP3A4 that patently increases circulating levels of some other HMG-CoA reductase inhibitors [20].

The main objective of the current study was to present a further example which showed a victorious use of an advanced mathematical modeling method based on the concept of a dynamic system in mathematical modeling in pharmacokinetics [3-14]. An additional objective was to motivate researchers in pharmacokinetics to use of an alternative modeling method to those modeling methods commonly used in pharmacokinetic studies. Previous examples presenting an advantageous use of the modeling method used in the current study can be found in full-text articles available completely free of cost on the authors' web pages at: http://www.uef.sav.sk/durisova.htm and http:// www.uef.sav.sk/advanced.htm.

http://www.slovaklines.sk/fileadmin/user_upload/ cestovne_poriadky/pal/102426.pdf

\section{Methods}

The data published in the study [1] were employed. For modeling purposes, an advanced mathematical modeling method modeling method based on the concept of a dynamic system was used; see e.g. the studies cited above. The development of a mathematical model of each dynamic system $H$ was based on the following simplifying assumptions: a) initial conditions of each dynamic system $H$ be zero; b) pharmacokinetic processes occurring in the body once oral erythromycin administration; were linear and time-invariant, c) concentrations of erythromycin were the same throughout all subsystems of the dynamic systems $H$ (where subsystems were integral parts of whole dynamic systems $H$ ); d) no barriers to the distribution and/or elimination of erythromycin existed. The modeling process used in the present study can be described as follows:

In the first step of the method, a dynamic system $H$, was defined for each volunteer by relating the Laplace transform of the serum concentration time profile of erythromycin, denoted $C(s)$, and the Laplace transform of the erythromycin oral input into the body, denoted $I(S)$.

In the second step of the method, the dynamic systems $H$, were used to mathematically represent dynamic relations between erythromycin inputs into the body and erythromycin behavior in the body [14-16]. 
In the third step of the method, the transfer function, denoted $H(s)$, (see Eq. 1) was derived for each active system $H$ by relating Laplace transform of the mathematical illustration of the serum concentration-versus-time profile of erythromycin denoted $C(\mathrm{~s})$, and the Laplace transform of the mathematical illustration of the oral administration of erythromycin, denoted $I(s)$, (the lower case letter " $s$ " denotes the complex Laplace variable), see e.g. the studies cited above and the following equation:

$$
H(s)=\frac{C(s)}{I(s)} .
$$

Thereafter, the dynamic system $H$ of each volunteer was described with transfer function denoted $H(s)$, see, e.g. the following studies [3-14] and references therein.

For modeling purpose, the software named CTDB [8] and transmit the function model $H_{M}(s)$, described in the following equation were used:

$$
H_{M}(s)=G \frac{a_{0}+a_{1} s+\ldots+a_{n} s^{n}}{1+b_{1} s+\ldots+b_{m} s^{m}} .
$$

On the right-hand-side of Eq. [2] is filling a approximant of $H_{M}(s)$ [19], $G$ is an estimator of the model parameter known as the gain of the dynamic system $H, a_{1}, \ldots a_{\mathrm{n}}, b_{1}, \ldots b_{\mathrm{m}}$ are the additional model parameters, $n$ is the maximum degree of the nominator polynomial, and $m$ is the maximum degree of the denominator polynomial, where $n<m$, see e.g. the following studies [3-14].

In the fourth step of the method, the transfer function $H(s)$ was converted into equivalent frequency response function, denoted $F\left(i \omega_{j}\right)$ [14].

In the fifth step of the method, the non-iterative method published earlier [3-14] was used to determine a mathematical model of the frequency response function $F_{M}\left(i \omega_{j}\right)$ and point estimates the parameters of the model frequency response function $F_{M}\left(i \omega_{j}\right)$ in the complex domain for each volunteer. The model of the frequency response function $F_{M}\left(i \omega_{j}\right)$ used in the recent study is described by the following equation:

$$
F_{M}\left(i \omega_{j}\right)=G \frac{a_{0}+a_{1} i \omega_{j}+\ldots+a_{n}(i \omega)^{n}}{1+b_{1} i \omega_{j}+\ldots+b_{m}\left(i \omega_{j}\right)^{m}} .
$$

Analogously as in Eq. [2]: $n$ is the maximum degree of the numerator polynomial of the model frequency response function $F_{M}\left(i \omega_{j}\right), m$ is the maximum degree of the denominator polynomial of the model frequency response function $F_{M}\left(i \omega_{j}\right)$, $n \leq m, . i$ is the invented unit, and $\omega$ is the angular frequency in Eq.(3). In the fifth step of the method, each the model frequency response function $F_{M}\left(i \omega_{j}\right)$ was advanced, using the Monte-Carlo and the Gauss-Newton method in the time domain.

In the sixth step of the method, the Akaike information was used to differentiate among models of frequency response functions $F_{M}\left(i \omega_{j}\right)$ of dissimilar complication and to select the best model of the frequency response function $F_{M}\left(i \omega_{j}\right)$ with the minimum value of the Akaike information criterion [15]. In the final step of the method, $95 \%$ confidence intervals for parameters of the final models $F_{M}\left(i \omega_{j}\right)$ were determined.
After the improvement of mathematical models of the dynamic systems $H$, the following primary pharmacokinetic variables were determined: The time occurrence of the maximum observed plasma concentration of erythromycin, denoted $t_{\text {max }}$ the maximum observed plasma concentration of erythromycin, denoted $C_{m a x}$ the elimination half-time of erythromycin, denoted $t_{1 / 2}$ area under the plasma concentration versus time profile of erythromycin from time zero to infinity, denoted, $A U C_{o-\infty}$, and total body clearance of erythromycin, denoted by $C I$.

The transfer function model $H_{M}(s)$ and the frequency response function model $F_{M}\left(i \omega_{j}\right)$ are implemented in the computer program CTDB [8]. A sample version of the computer program CTDB is available at: http://www.uef.sav.sk/advanced. $\mathrm{htm}$. Transfer functions and frequency response functions are not unknown in pharmacokinetics, see e.g. the following studies $[20,21]$.

\section{Results}

The best-fit third-order model of $F_{M}\left(i \omega_{j}\right)$ selected with the Akaike information criterion was described by Eq. (4):

$$
F_{M}\left(i \omega_{j}\right)=G \frac{a_{0}+a_{1} i \omega_{j}}{1+b_{1} i \omega_{j}+b_{2} i \omega_{2}+b_{3} i \omega_{3}} .
$$

This model provided an adequate fit to the erythromycin concentration data in all volunteers investigated in the previous [1] and the current study. Estimates of the model parameters $a_{0}$, $a_{1}, a_{2}, a_{3}$ are listed in Table 1. Model-based estimates of primary pharmacokinetic variables were listed in Table 2.

Volunteer No.1 was arbitrarily chosen from fourteen volunteers investigated in the previous study [1] and in the current study, to illustrate the results obtained. Figure 1 showed the experimental plasma concentration versus time profile of erythromycin and the report of the observed profile with the developed model of the dynamic system $H$. Analogous results also hold for all volunteers participating in the previous [1] and the current study.

\section{Discussion}

The dynamic systems used in the current study were mathematical objects, without any physiological application. They were used to model dynamic relationships between

Table 1: Parameters of the third-order model of the dynamic system describing the pharmacokinetic behavior of orally administered erythromycin to volunteer No.1.

\begin{tabular}{|l|l|l|}
\hline Model parameters & $\begin{array}{l}\text { Estimates of } \\
\text { model parameters }\end{array}$ & (95\% CI) \\
\hline G $\left({\left.\mathrm{h} . \mathrm{I}^{-1}\right)}^{\text {erythromycin to volunt }}\right.$ & 0.007 & 0.006 to 0.012 \\
\hline$a_{0}(-)$ & 0.99 & 0.81 to 1.02 \\
\hline$a_{1}(\mathrm{~min})$ & 59.15 & 48.12 to 62.38 \\
\hline$b_{1}(\mathrm{~min})$ & 461.88 & 460.73 ton 472.02 \\
\hline$b_{2}\left(\min ^{2}\right)$ & 6033.61 & 6028.59 to 6040.33 \\
\hline$b_{3}\left(\min ^{3}\right)$ & 3678275.74 & $\begin{array}{l}3678271.05 \\
3678280.33\end{array}$ \\
\hline
\end{tabular}


Table 2: Pharmacokinetic variables of orally administered erythromycin to volunteer No.1.

\begin{tabular}{|l|l|}
\hline Pharmacokinetic variable & Estimates \\
\hline $\begin{array}{l}\text { The time of the maximum } \\
\text { observed concentration of erythromycin } \\
\mathrm{t}_{\text {max }}(\mathrm{h})\end{array}$ & $3.12 \pm 0.61^{*}$ \\
\hline $\begin{array}{l}\text { The maximum observed concentration of MLP } \\
\text { erythromycin } \mathrm{m}_{\text {max }}(\mu \mathrm{grj} / \mathrm{ml})\end{array}$ & $293,1 \pm 18.52$ \\
\hline $\begin{array}{l}\text { The plasma elimination half-time of erythromycin } t_{1 / 2} \\
\text { (hod) }\end{array}$ & $2.4 \pm 0.41$ \\
\hline Renal clearance of erythromycin /F (L/hr) & $12.2 \pm 15.25$ \\
\hline Mean absorption time of erythromycin $(\mathrm{hr})$ & $0.84 \pm 0.1$ \\
\hline $\begin{array}{l}\text { Men residence time of erythromycin } \\
\left.\text { CI (ml.min }{ }^{-1}\right) \text { from plasma }\end{array}$ & $3.67 \pm 5.06$ \\
\hline standard deviation & \\
\hline
\end{tabular}

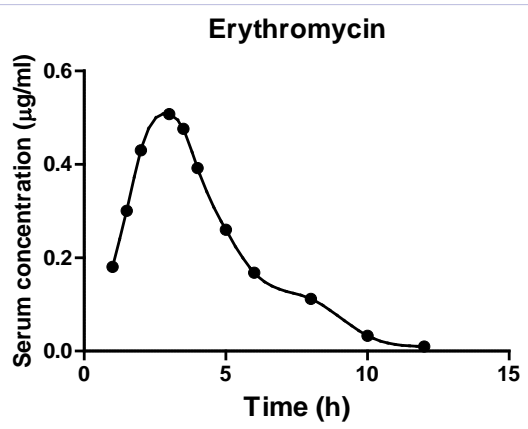

Figure 1: Observed serum concentration versus time profile of erythromycin and the description of the observed profile with the model of the volunteer's No.1 dynamic system which mathematically represented the dynamic relation between erythromycin input to the body and behavior of erythromycin in volunteer No. 1.

Table 3: Observed and model predicted blood concentrations of erythromycin in volunteer No.1.

\begin{tabular}{|c|c|c|}
\hline Time (h) & $\begin{array}{c}\text { Observed } \\
\text { concentration of } \\
\text { erythromycin (ng/ } \\
\text { ml) }\end{array}$ & $\begin{array}{c}\text { Model predicted } \\
\text { concentration of } \\
\text { erythromycin (ng/ } \\
\text { ml) }\end{array}$ \\
\hline 0 & 0 & 0 \\
\hline 0,5 & 47.4 & 48 \\
\hline 1.0 & 185 & 185 \\
\hline 2 & 293 & 293 \\
\hline 4 & 254 & 254 \\
\hline 6 & 158 & 158 \\
\hline 9 & 76.9 & 76.9 \\
\hline 12 & 130 & 130 \\
\hline
\end{tabular}

erythromycin inputs into the body [15-17] and behavior of erythromycin in the body. The method used in the recent study has been described in detail in the previous studies [3-13].

As in previous studies, authored or co-authored by the author of the recent study, the development of mathematical models of the dynamic systems $H$, was based on the known inputs and outputs of the dynamic systems $H$. In general, a dynamic system is modeled using the transfer function models $H_{M}(s)$, as it was the case in the recent study, then the accuracy of the model depends in large part of the degrees of polynomials of the transfer function models $H_{M}(s)$ used to fit the data, see e.g. the following studies [3-14].

The parameter gain is also known as gain coefficient, or gain factor. Generally, the parameter gain is defined as relationship between the magnitudes of an output of the dynamic system to a magnitude of an input to the dynamic system in steady state. Or in other words, the parameter gain of a dynamic system is a proportional value that shows the relationship between the magnitudes of an output to a magnitude of an input of a dynamic system in steady state.

The pharmacokinetic meaning of the parameter gain depends on the nature of the dynamic system; see e.g. studies available at: http://www.uef.sav.sk/advanced.htm.

The non-iterative method published in the study [14] and used in the recent study is capable of providing quick identification of an optimal structure of a model frequency response. This is a great advantage of this method, because this significantly speeds up the development of frequency response models. The reason for conversion of $H_{M}(s)$ to $F_{M}\left(i \omega_{j}\right)$ was as explained in the following text. The variable: "s" in $H_{M}(s)$ is a complex Laplace variable (see Eq. [2]), while the angular frequency $\omega$ (see Eq. [4]) is a real variable, what is suitable for modeling purposes.

The mathematical models developed in the recent study sufficiently approximated the dynamic relationships between erythromycin input to the body and behavior of erythromycin in the body in the volunteers investigated in the previous [1] and the current study.

The current study again showed that mathematical and computational tools from system engineering can be successfully used in mathematical modeling in pharmacokinetics. Frequency response functions are complex functions, therefore modeling is performed in the complex domain. In addition, the modeling methods used to develop model frequency response functions are computationally intensive, and modeling require as a minimum partial knowledge of the theory of dynamic system, and an abstract way of thinking about the dynamic system under study.

The principle difference between pharmacokinetic modeling methods traditionally used in pharmacokinetic studies and modeling methods that use mathematical and computational tools from the theory of dynamic systems, is as follows: the former methods are based on modeling plasma (or blood) concentrationtime profiles of drugs, however the latter methods are based on modeling dynamic relationships between a mathematically represented drug inputs into the body and mathematically represent resulting plasma (or blood) concentration-time profiles of administered drugs. See e.g. the previous studies authored and/or coauthored by the author of the current study and the explanatory example, available free of cost at the author's Web page: http://www.uef.sav.sk/advanced.htm. 
The computational and modeling methods that use computational and modeling tools from the theory of dynamic systems that can be example for adjustment of drug administration aimed at achieving and then maintaining required drug concentration-time profiles in patients see e.g. the following study [6]. The methods considered here can be used for safe and cost-effective individualization of drug dosing e.g. through computer-controlled infusion pumps. This is important example for administration of clotting factors to hemophilia patients, as exemplified in the study [6].

The advantages of the model and modeling method used in the recent study are evident here: The models developed and used overcome one of the typical limitations of compartmental models: For the development and use of the models considered here, an assumption of well-mixed spaces in the body is not necessary. The basic structures of the models developed are used largely applicable to mathematical modeling different dynamic systems in the field of pharmacokinetics and in many other scientific as well as practical fields. From a point of view pharmacokinetic community, is an advantage of the models developed using computational tools from the theory of dynamic systems is that the models considered here emphasize dynamic relationships between drug inputs into the body and behavior of a drug in a human and/or an animal body. The method used in the current study can be easily generalized. Therefore, there is no problem to use the method considered here in several scientific and practical fields. Transfer functions of dynamic systems are not unknown in pharmacokinetics; see e.g. the following studies [20-22].

\section{Conclusion}

The models developed and used in the current study are successfully described the pharmacokinetic behavior of oral administration to healthy male adult volunteers. The modeling method used is universal, comprehensive and flexible and thus it can be applied to a broad range of dynamic systems in the field of pharmacokinetics and in many other fields. The current study repeatedly presented an attempt to visualize the successful use of mathematical and computational tools from the theory of dynamic systems in pharmacokinetic modeling. For the previous attempts with the use of the modeling method used in the current study please visit http://www.uef.sav.sk/advanced. htm. The current study repeatedly showed that an integration of pharmacokinetic and bioengineering approaches is a good and efficient way to study processes in pharmacokinetics, because for this is that such integration combines mathematical rigor with biological insight.

\section{References}

1. Yakatan GJ, Poynor WJ, Harris RG, Martin A, Leonard RG, Briggs RG et al. Single-dose fasting bioequivalence assessment of erythromycin stearate tablets in man. J Pharmacokin Biopharm. 1979;7(4):355-368.

2. Cooper KJ, Martin PD, Dane AL, Warwick MJ, Raza A, Schneck DW. The effect of erythromycin on the pharmacokinetics of rosuvastatin. Eur Clin Pharmacol. 2003;59(1):51-56.

3. van Rossum JM, de Bie JE, van Lingen G, Teeuwen HW
Pharmacokinetics from a dynamical systems point of view. Clin Pharmacokinet. 1989;17:27-44.

4. Dedik L, Ďurišová M. Frequency response method in pharmacokinetics. 1994;22(4)293-307.

5. Dedik L, Ďurišová M. CXT-MAIN: A software package for the determination of the analytical form of the pharmacokinetic system weighting function. Comput Methods Programs Biomed. 1996;51(3):183-192.

6. Ďurišová M, Dedík L. Modeling in frequency domain used for assessment of in vivo dissolution profile. Pharm Res. 1997;14(7):860864 .

7. Ďurišová M, Dedík L. A system-approach method for the adjustment of time-varying continuous drug infusion in individual patients: A simulation study. J Pharmacokinet Pharmacodyn. 2002;29(5-6):427444 .

8. Ďurišová M, Dedík L. New mathematical methods in pharmacokinetic modeling. Basic Clin. Pharmacol Toxicol. 2005;96(5):335-342.

9. Dedík L, Ďurišová M, Penesová A, Miklovičová D, Tvrdoňová M. Estimation of influence of gastric emptying on shape of glucose concentration-time profile measured in oral glucose tolerance test. Diabetes Res Clin Pract. 2007;77(3):377-384.

10. Ďurišová M. A physiological view and structures of mean residence times. Gen. Physiol. Biophys. 2014;33(1):75-80. doi: 10.4149/ gpb_2013060.

11.Ďurišová M. Mathematical Model of Pharmacokinetic Behavior of Orally Administered Prednisolone in Healthy Volunteers. J Pharmaceu Pharmacol. 2014;2(2):1-5

12.Ďurišová M. Further worked out examples that illustrated the successful use of an advanced mathematical modeling method based on the theory of dynamic systems in pharmacokinetics. Int. J Res Sci Res. 2015;6(6):4873-4879.

13. Ďurišová M, Dedík L, Kristová V, Vojtko R. Mathematical model indicates nonlinearity of noradrenaline effect on rat renal artery. Physiol Res. 2008;57(5):785-788.

14. Ďurišová M. A physiological view and structures of mean residence times. Gen Physiol Biophys. 2014;33(1):75-80. doi: 10.4149/ gpb_2013060.

15. Weiss M, Pang, KS. Dynamics of drug distribution. The role of the second and third curve moments. J Pharmacokinet Biopharm. 1992;20(3):253-278.

16. Verotta, D. Concepts, properties, and applications of linear systems to describe distribution, identify inputs, and control endogenous substances and drugs in biological systems. Crit Rev Biomed Eng. 1996;24(2-3):73-139.

17. Xiao H, Yang Q, Cai H, Qi R, Yan L, Liu S, et al. A prodrug strategy to deliver cisplatin (IV) and paclitaxel in nanomicelles to improve efficacy and tolerance. Biomaterials. 2012;33(27):6507-6519. doi: 10.1016/j.biomaterials.2012.05.049.

18. Levy EC. Complex curve fitting. IRE Transactions on Automatic Control. 1959;4(1):37-43. DOI:10.1109/TAC.1959.6429401 http://ieeexplore.ieee.org/xpl/articleDetails.jsp?arnumber $=6429401$

19. Akaike H. A new look at the statistical model identification. IEEE Trans Automat Contr. 1974;19(6):716-723. DOI: 10.1109/ TAC.1974.1100705. http://ieeexplore.ieee.org/xpl/login. jsp?tp=\&arnumber=1100705 
20. Hargreaves G, Higham NJ. Efficient algorithms for the matrix cosine and sine. Num Algorithm. 2005;40:383-400. DOI 10.1007/s11075005-8141-0

21. Siegel RA. Pharmacokinetic transfer functions and generalized clearances. J Pharmacokin Biopharm. 1986;14(5):511-521.

22. Segre G. The sojourn time and its prospective use in pharmacology. J Pharmacokinet Biopharm. 1988;16(6):657-666.
23. Yates JW. Structural identifiability of physiologically based pharmacokinetic models. J Pharmacokinet Pharmacodyn. 2006;33(4):421-439.

24. Rescigno A. Compartmental analysis and its manifold applications to pharmacokinetics. AAPS Journal. 2010;12(1):61-72. doi: 10.1208/ s12248-009-9160-x. 